\title{
NAMPT promotes hepatitis B virus replication and liver cancer cell proliferation through the regulation of aerobic glycolysis
}

\author{
HUI-JIE GUO $^{1 *}$, HONG-YU LI ${ }^{1 *}$, ZI-HAO CHEN ${ }^{1 *}$, WEN-JING ZHOU ${ }^{1}$, JIA-JIE LI ${ }^{1}$, JIA-YI ZHANG ${ }^{1}$, \\ JING WANG ${ }^{1}$, XING-YAN LUO ${ }^{1}$, TING ZENG ${ }^{1}$, ZHAO SHI $^{2}$ and CHUN-FEN MO ${ }^{1}$ \\ ${ }^{1}$ Department of Immunology, School of Basic Medical Sciences, Chengdu Medical College; \\ ${ }^{2}$ Department of Anatomy, Histology and Embryology, Development and Regeneration Key Laboratory \\ of Sichuan Province, Chengdu Medical College, Chengdu, Sichuan 610500, P.R. China
}

Received July 29, 2020; Accepted January 11, 2021

DOI: $10.3892 / 01.2021 .12651$

\begin{abstract}
Nicotinamide phosphoribosyltransferase (NAMPT) is a critical rate-limiting enzyme involved in NAD synthesis that has been shown to contribute to the progression of liver cancer. However, the potential role and mechanism of NAMPT in hepatitis B virus (HBV)-associated liver cancer remain unclear. The present study assessed the expression of NAMPT in HBV-positive and -negative liver cancer cells, and investigated whether HBV-induced NAMPT expression is dependent on HBV X protein (HBx). In addition, the role of NAMPT in HBV replication and transcription, and in HBV-mediated liver cancer cell growth was explored. The effects of NAMPT on the glycolytic pathway were also evaluated. Reverse transcription-quantitative PCR and western blotting results revealed that NAMPT expression levels were significantly higher in HBV-positive liver cancer cells than in HBV-negative liver cancer cells, and this effect was HBx-dependent. Moreover, the activation of NAMPT was demonstrated to be required for HBV replication and transcription. The NAMPT inhibitor FK866 repressed cell survival and promoted cell death in $\mathrm{HBV}$-expressing liver cancer cells, and these effects were attenuated by nicotinamide mononucleotide. Furthermore, the inhibition of NAMPT was associated with decreased glucose uptake, decreased lactate production and decreased ATP
\end{abstract}

Correspondence to: Dr Chun-Fen Mo, Department of Immunology, School of Basic Medical Sciences, Chengdu Medical College, 783 Xindu Avenue, Chengdu, Sichuan 610500, P.R. China E-mail: mochunfen@cmc.edu.cn

Dr Zhao Shi, Department of Anatomy, Histology and Embryology, Development and Regeneration Key Laboratory of Sichuan Province, Chengdu Medical College, 783 Xindu Avenue, Chengdu, Sichuan 610500, P.R. China

E-mail: freenemo@126.com

${ }^{*}$ Contributed equally

Key words: NAMPT, hepatitis B virus, liver cancer, aerobic glycolysis levels in HBV-expressing liver cancer cells, indicating that NAMPT may promote the aerobic glycolysis. Collectively, these findings reveal a positive feedback loop in which HBV enhances NAMPT expression and the activation of NAMPT promotes HBV replication and HBV-mediated malignant cell growth in liver cancer. The present study highlights the important role of NAMPT in the regulation of aerobic glycolysis in HBV-mediated liver cancer, and suggests that NAMPT may be a promising treatment target for patients with HBV-associated liver cancer.

\section{Introduction}

Liver cancer is one of the most frequently occurring malignancies and the second leading cause of cancer-associated mortality worldwide (1). Hepatitis B virus (HBV) infection is a global health concern that increases the risk of liver cancer 5-fold (2). Epidemiological studies have reported that 240 million individuals are infected with HBV and approximately 650,000 succumb to $\mathrm{HBV}$-associated complications each year (3). The HBV genome encodes four main proteins, denoted as $\mathrm{C}, \mathrm{P}, \mathrm{S}$ and $\mathrm{X}$. Accumulating evidence indicates that $\mathrm{HBV} \mathrm{X}$ protein $(\mathrm{HBx})$ plays a crucial role in $\mathrm{HBV}$-mediated carcinogenesis and liver cancer progression through the direct or indirect regulation of host gene transcription and protein activity $(4,5)$. Therefore, a better understanding of the molecular mechanisms underlying HBV-mediated liver cancer pathogenesis may improve the diagnosis and treatment of this disease.

Nicotinamide phosphoribosyltransferase (NAMPT) is a critical rate-limiting enzyme involved in NAD synthesis that catalyzes the conversion of nicotinamide (NAM) to nicotinamide mononucleotide (NMN). Since NAD acts as a co-enzyme in the regulation of various metabolic signaling pathways, including glycolysis, serine biosynthesis and fatty acid oxidation, the continuous generation of NAD promotes the survival and proliferation of cancer cells $(6,7)$. Previous studies have shown that NAMPT expression is upregulated in different types of cancer, including breast, lung and prostate cancer $(8,9)$. Therefore, the inhibition of NAMPT represents a potential therapeutic strategy for killing cancer cells. The specific NAMPT inhibitor FK866 has been shown to 
exhibit potent anticancer activity in several types of cancer, including gastric, pancreatic cancer and chronic lymphocytic leukemia (10-12). However, whether NAMPT is involved in $\mathrm{HBV}$ replication and $\mathrm{HBV}$-mediated liver cancer progression remains unknown.

In the present study, the expression levels of NAMPT were assessed in HBV-positive and HBV-negative liver cancer cells, and whether HBV-induced NAMPT expression was $\mathrm{HBx}$-dependent was investigated. In addition, the role of NAMPT in HBV replication and transcription, as well as HBV-mediated liver cancer cell growth was explored. Moreover, the effects of NAMPT on the glycolytic pathway of HBV-expressing liver cancer cells were evaluated.

\section{Materials and methods}

Cell culture and reagents. HBV negative liver cancer cells (HepG2 and Huh7) were purchased from the Chinese Academy of Sciences Cell Bank (Shanghai, China) and maintained in DMEM medium (HyClone; Cytiva) at $37^{\circ} \mathrm{C}$ in a $5 \% \mathrm{CO}_{2}$ incubator. The medium was supplemented with $10 \%$ FBS (Gibco; Thermo Fisher Scientific Inc.), $100 \mathrm{IU} / \mathrm{ml}$ penicillin and $100 \mathrm{IU} / \mathrm{ml}$ streptomycin. HBV positive liver cancer cells (HepG2.2.15 and HepAD38) cells were cultured in DMEM medium (HyClone; Cytiva) supplemented with $400 \mu \mathrm{g} / \mathrm{ml}$ G418 (Sangon Biotech Co., Ltd) at $37^{\circ} \mathrm{C}$ in a $5 \% \mathrm{CO}_{2}$ incubator as described in our previous study (13). The HepG2 and HepG2.2.15 cells were assessed by short tandem repeat (STR) analysis and no contamination with other cell lines was present. Moreover, STR analysis verified that the cell profile was consistent with that of the archived cells.

The following reagents were used: NAMPT inhibitor FK866 (S2799; Selleck Chemicals), NMN (S5259; Selleck Chemicals) and trypan blue (T6146; Sigma-Aldrich; Merck $\mathrm{KGaA}$ ). The cells were treated with $20 \mathrm{nM}$ FK866 alone or in combination with $500 \mu \mathrm{M} \mathrm{NMN}$ for $24 \mathrm{~h}$ at $37^{\circ} \mathrm{C}$.

Plasmid construction and cell transfection. The HBV1.3 plasmid harboring the HBV genome (subtype adw), the plasmid encoding HBx amplified from the HBV genome, and the HBV expression plasmid with an $\mathrm{HBx}$ mutation $(\triangle \mathrm{HBx})$ were generated as described previously (14). HepG2 and Huh7 cells were transfected with $4 \mu \mathrm{g}$ of HBV1.3, HBx, or $\Delta \mathrm{HBx}$ plasmid by using Lipofectamine ${ }^{\circledR} 3000$ (cat. no. L3000015; Invitrogen; Thermo Fisher Scientific, Inc.). HepG2.2.15 and HepAD38 cells were transfected with $100 \mathrm{nmol} / \mathrm{l}$ of specific NAMPT siRNAs (cat. no. sc-45843; Santa Cruz Biotechnology Inc.) or negative control siRNA (cat. no. sc-37007; Santa Cruz Biotechnology Inc.) by using Lipofectamine RNAiMAX (cat. no. 13778150; Thermo Fisher Scientific, Inc.). After transfection at $37^{\circ} \mathrm{C}$ for $48 \mathrm{~h}$, cells were harvested for subsequent experiments.

RNA extraction and reverse transcription-quantitative PCR $(R T-q P C R)$. Total RNA was extracted using from cells using TRIzol $^{\circledR}$ reagent (Invitrogen; Thermo Fisher Scientific, Inc.) and reverse transcribed to cDNA using the PrimeScript ${ }^{\mathrm{TM}}$ RT reagent Kit (Takara Bio, Inc.) according to the manufacturer's protocol. The relative levels of the target gene mRNA transcripts were determined by qPCR with SYBR ${ }^{\circledR}$ Premix Ex $\mathrm{Taq}^{\mathrm{TM}}$ II (Takara Bio, Inc.) using an iCycler $\mathrm{iQ}^{\mathrm{TM}}$ Multicolor
Real-Time PCR Detection System (Bio-Rad Laboratories, Inc.) as previously described (15). The thermocycling conditions were as follows: Initial denaturation at $95^{\circ} \mathrm{C}$ for $5 \mathrm{~min}$ followed by 40 cycles of $95^{\circ} \mathrm{C}$ for $15 \mathrm{sec}, 60^{\circ} \mathrm{C}$ for $20 \mathrm{sec}$, and $72^{\circ} \mathrm{C}$ for $30 \mathrm{sec}$ followed by final elongation at $72^{\circ} \mathrm{C}$ for $2 \mathrm{~min}$. The following primers were used: Glucose transporter 1 (GLUT1), 5'-CGGGCCAAGAGTGTGCTAAA-3' (forward) and 5'-TGA CGATACCGGAGCCAATG-3' (reverse); lactate dehydrogenase A (LDHA), 5'-GGCCTGTGCCATCAGTATCT-3' (forward) and 5'-GGAGATCCATCATCTCTCCC-3' (reverse); NAMPT, 5'-TACAAGTTGCTGCCACCTTATC-3' (forward) and 5'-GCAAACCTCCACCAGAACC-3' (reverse); pyruvate kinase M2 (PKM2), 5'-TGTCTGGAGAAACAGCCAAAG G-3' (forward) and 5'-CGGAGTTCCTCAAATAATTGC AA-3' (reverse); HBV, 5'-ACCGACCTTGAGGCATACTT-3' (forward) and 5'-GCCTACAGCCTCCTAGTACA-3' (reverse); HBV core antigen (HBcAg), 5'-CTGGGTGGGTGTTAATTT GG-3' (forward) and 5'-TAAGCTGGAGGAGTGCGAAT-3' (reverse); HBV surface antigen (HBsAg), 5'-CTCCAATCA CTCACCAACCT-3' (forward) and 5'-TCCAGAAGAACC AACAAGAAGA-3' (reverse); GAPDH, 5'-ACCACAGTCCAT GCCATCAC-3' (forward) and 5'-TCCACCACCCTGTTG CTGTA-3' (reverse). The $2^{-\triangle \Delta C \mathrm{Cq}}$ method was used in analyzing relative gene expression data (16).

Westernblotanalysis. The cells werelysed with RIPAlysisbuffer (50 mM Tris- $\mathrm{HCl}$, pH 7.5, $150 \mathrm{mM} \mathrm{NaCl}, 1 \%$ Triton X-100, $0.1 \%$ SDS, $0.5 \%$ deoxycolic acid) containing protease inhibitors (Complete Protease Inhibitor Cocktail Tablets; Roche Diagnostics) for $30 \mathrm{~min}$ on ice. The concentration of extracted proteins were determined by Bradford method. Cell lysates (40 $\mu \mathrm{g} /$ lane) were resolved by $12 \%$ SDS-PAGE and transferred to PVDF membranes (EMD Millipore). After blocking with $5 \%$ skimmed milk at room temperature for $1 \mathrm{~h}$, the membrane was incubated overnight at $4^{\circ} \mathrm{C}$ with the following primary antibodies: GLUT1 (1:1,000; cat. no. 12939; Cell Signaling Technology, Inc.), LDHA (1:1,000; cat. no. 3582; Cell Signaling Technology, Inc.), NAMPT (1:1,000; cat. no. ab45890; Abcam), PKM2 (1:1,000; cat. no. 4053; Cell Signaling Technology, Inc.), HBx (1:1,000; cat. no. MA1-081; Thermo Fisher Scientific, Inc.) and $\beta$-actin (1:2,000; cat. no. sc-47778; Santa Cruz Biotechnology, Inc.). After washing with TBS with $0.1 \%$ Tween-20 (TBST) three times, the membranes were subsequently incubated with HRP-conjugated secondary antibody (1:3,000; cat. nos. sc-2004 and sc-2005; Santa Cruz Biotechnology, Inc.) at room temperature for $1 \mathrm{~h}$. Following three washes with TBST, the membrane was visualized using an Immobilon ${ }^{\mathrm{TM}}$ Western Chemiluminescent HRP substrate (EMD Millipore).

Determination of HBsAg, HBeAg and HBV DNA. The quantities of HBsAg and $\mathrm{HBeAg}$ in the cell supernatant were measured using ELISA kits (Kehua Bio-Engineering Co., Ltd.) and the quantity of HBV DNA in the cell-free culture medium was detected using the $\operatorname{artus}{ }^{\circledR}$ HBV RG PCR Kit (Qiagen AB) according to the manufacturer's instructions.

Measurement of cell viability. Cell viability analysis was performed using a Cell Counting Kit-8 assay (CCK-8; KN867; Dojindo Molecular Technologies, Inc.) as previously 
described (17). Briefly, the cells were plated in 96-well plates (1x10 4 cells/well) and treated with $20 \mathrm{nM}$ FK866 alone or in combination with $500 \mu \mathrm{M} \mathrm{NMN}$ for $24 \mathrm{~h}$ at $37^{\circ} \mathrm{C}$. After this, $10 \mu \mathrm{l}$ CCK-8 solution was added to each well and the plate was incubated for an additional $4 \mathrm{~h}$. The absorbance was then measured at $450 \mathrm{~nm}$ using a microplate reader. For trypan blue exclusion assay, $2 \times 10^{5}$ cells were plated in 6 -well plates and treated with $20 \mathrm{nM}$ FK866 alone or in combination with $500 \mu \mathrm{M} \mathrm{NMN}$ for $24 \mathrm{~h}$ at $37^{\circ} \mathrm{C}$. Then, the cells were digested with trypsin and resuspended in PBS containing 0.04\% trypan blue. The viable cells (clear cytoplasm) and death cells (blue cytoplasm) were counted under a light microscope.

Colony formation assay. Cells were plated into 24-well-plates (500 cells/well) and subsequently treated with $20 \mathrm{nM}$ FK866 alone or in combination with $500 \mu \mathrm{M} \mathrm{NMN}$ at $37^{\circ} \mathrm{C}$. The colonies were allowed to grow for $\sim 10$ days, and then fixed in $4 \%$ paraformaldehyde at room temperature for $15 \mathrm{~min}$ and stained with crystal violet at room temperature for $20 \mathrm{~min}$. The number of colonies was counted using ImagJ software (v.1.52a; National Institutes of Health).

Glycolysis examination. The extracellular acidification rate (ECAR) of the cells was determined using a Seahorse XF Glycolysis Stress Test Kit with an XF96 Extracellular Flux Analyzer (Seahorse Bioscience; Agilent Technologies, Inc.) according to the manufacturer's instructions. Briefly, $2 \times 10^{4}$ cells were plated into XF96 cell plates (Agilent Technologies, Inc.) and treated with $20 \mathrm{nM}$ FK866 alone or in combination with $500 \mu \mathrm{M} \mathrm{NMN}$ at $37^{\circ} \mathrm{C}$ for $24 \mathrm{~h}$. After washing with XF assay media, each well of the XF96 cartridge was sequentially injected with glucose (detection of glycolysis), oligomycin (an ATPase inhibitor, which restrains mitochondrial ATP production) and 2-DG (a glucose analog, which inhibits glycolysis). Glucose uptake, lactate production and ATP concentration were measured in cell lysates using a Glucose Uptake Colorimetric Assay Kit (BioVision, Inc.), Lactate Colorimetric/Fluorometric Assay Kit (BioVision, Inc.) and ATP Colorimetric/Fluorometric Assay Kit (BioVision, Inc), respectively. The enzymatic activities of PKM2 and LDHA were determined using a Pyruvate Kinase Activity Assay Kit (Beijing Solarbio Science \& Technology Co., Ltd.) and Lactate Dehydrogenase Activity Colorimetric Assay Kit (BioVision, Inc.), respectively. All measurements were performed according to the manufacturer's protocols and normalized to the cell protein levels.

Statistical analysis. All quantitative data are expressed as the mean \pm SD. Comparisons between two groups were performed using the Student's t-test. Differences in quantitative data among multiple groups were analyzed by one-way ANOVA followed by Tukey's post hoc test. Data were analyzed using SPSS statistical software (version 22.0; IBM Corp.). $\mathrm{P}<0.05$ was considered to indicate a statistically significant difference.

\section{Results}

NAMPT expression levels are upregulated by HBx. To explore the association between NAMPT and HBV replication, the expression levels of NAMPT were assessed in HBV-producing liver cancer cells. The mRNA and protein expression levels of NAMPT were upregulated in HepG2.2.15 cells compared with those in HepG2 cells (Fig. 1A). In addition, elevated NAMPT levels were observed in untreated HepAD38 cells compared with tetracycline-treated HepAD38 cells (Fig. 1B). HepG2 and Huh7 cells transiently transfected with HBV-expressing plasmid HBV1.3 (harboring a 1.3-fold overlength of the HBV genome) demonstrated increased expression of NAMPT compared with that in cells transfected with empty vector (Fig. 1C and D). These data indicate that HBV replication promoted NAMPT expression in the liver cancer cells. To further assess whether HBV-induced NAMPT expression was $\mathrm{HBX}$-dependent, the $\triangle \mathrm{HBx}$ plasmid (HBV1.3 plasmid with HBx mutation) was transiently transfected into HepG2 and Huh7 cells and its effect on NAMPT expression was evaluated. As shown in Fig. 1C, the ectopic introduction of HBV1.3 plasmid but not that of $\triangle \mathrm{HBx}$ plasmid, significantly increased the expression levels of HBx in HepG2 cells. Notably, the mutation of HBx significantly mitigated the elevation in the levels of NAMPT induced by HBV replication in HepG2 cells. A similar result was observed in Huh7 cells (Fig. 1D). When the HBx expression plasmid was successfully introduced into HepG2 and Huh7 cells, the mRNA and protein expression levels of NAMPT were increased in response to the $\mathrm{HBx}$ stimulus (Fig. 1E and F). Therefore, these results indicate that HBx enhances the expression levels of NAMPT in liver cancer cells.

NAMPT activity is required for $H B V$ replication and transcription. To investigate the involvement of NAMPT in HBV replication, the extracellular levels of HBV DNA were assessed in HBV-producing liver cancer cells treated with the NAMPT inhibitor FK866. Extracellular HBV DNA levels were significantly reduced in HepG2.2.15 and HepAD38 cells following incubation with FK866 (Fig. 2A). Moreover, FK866 treatment resulted in a 2-fold reduction in HBV transcription (Fig. 2B), suggesting that NAMPT activity upregulates HBV production. To further verify the association between NAMPT activation and HBV replication, the expression levels of $\mathrm{HBcAg}$ and $\mathrm{HBsAg}$, which are indicators of active HBV replication, were assessed. The mRNA levels of $\mathrm{HBcAg}$ and HBsAg in FK866-treated HepG2.2.15 and HepAD38 cells were lower than those observed in vehicle-treated control cells (Fig. 2C and D). In addition, ELISAs indicated that the inhibition of NAMPT activity induced a significant reduction in the secretion of HBeAg and HBsAg into the supernatant of HepG2.2.15 and HepAD38 cells (Fig. 2E and F). Collectively, these data imply that the activation of NAMPT is essential for HBV replication and transcription.

NAMPT inhibition by FK866 represses $H B V$-mediated cell survival. To further investigate the effect of NAMPT on $\mathrm{HBV}$-mediated liver cancer cell growth, liver cancer cells were treated with FK866 and cell proliferation was assessed using a CCK-8 assay. Treatment with FK866 resulted in a significant reduction in the viability of HBV-producing liver cancer cells, whereas the exogenous addition of NMN attenuated the FK866-induced cytotoxicity. Consistent with the CCK-8 assay results, the number of colonies formed by FK866-treated HepG2.2.15 and HepAD38 cells was significantly lower 
A

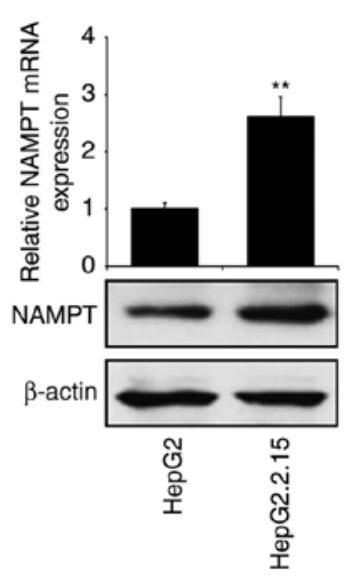

D

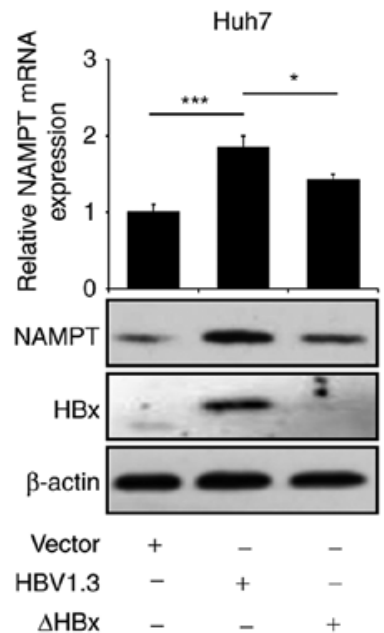

B

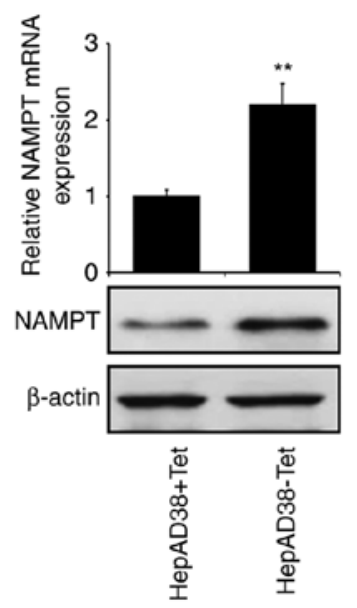

E

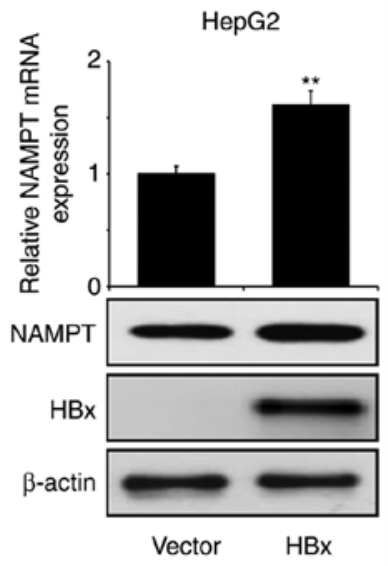

C

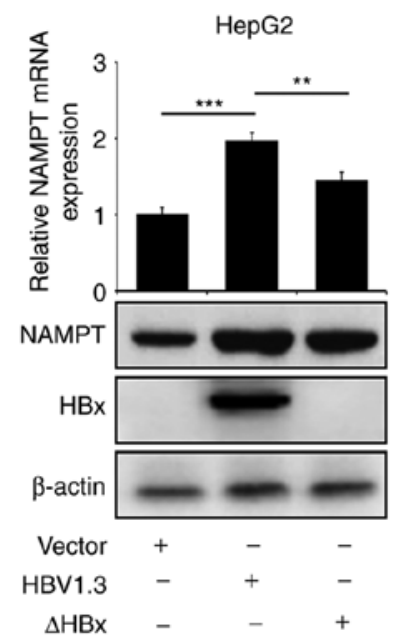

$\mathrm{F}$

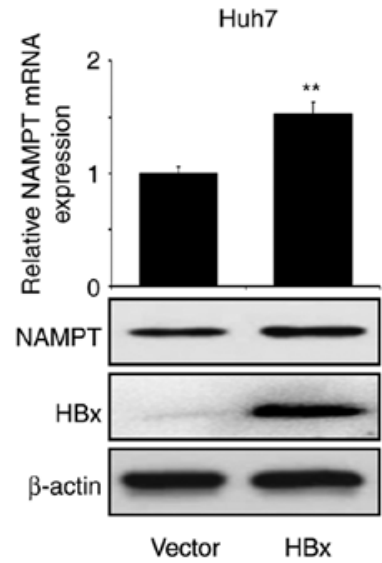

Figure 1. HBx increases NAMPT expression in liver cancer cells. (A) RT-qPCR and western blot analyses of NAMPT expression were performed in (A) HepG2 and HepG2.2.15 cells and (B) HepAD38 cells with or without Tet treatment. RT-qPCR and western blot analyses of NAMPT expression in (C) HepG2 and (D) Huh7 cells transfected with HBV1.3 or $\triangle$ HBx plasmid. RT-qPCR and western blot analysis of NAMPT expression in (E) HepG2 and (F) Huh7 cells transfected with HBx plasmid. ${ }^{*} \mathrm{P}<0.05,{ }^{* * *} \mathrm{P}<0.01,{ }^{* * *} \mathrm{P}<0.001$. HBx, hepatitis B X protein; NAMPT, nicotinamide phosphoribosyltransferase; Tet, tetracycline; RT-qPCR, reverse transcription-quantitative PCR.

compared with that of vehicle-treated cells, while the co-administration of NMN rescued the colony formation of HepG2.2.15 and HepAD38 cells following FK866 supplementation (Fig. 3B). Furthermore, the inhibition of NAMPT by FK866 induced an increase in the death rate of HBV-expressing liver cancer cells that was reversed by NMN treatment (Fig. 3C). In addition, FK866 reduced markedly the protein expression level of $\mathrm{Bcl} 2$ and increased the levels of cleaved caspase-3, cleaved PARP and Bax (Fig. 3D). Co-treatment of the cells with NMN alleviated the FK866-induced effects on the expression of apoptosis-associated proteins (Fig. 3D). Therefore, these findings indicate that NAMPT contributes to the HBV-mediated malignant transformation of liver cancer.

NAMPT regulates aerobic glycolysis in $H B V$-expressing liver cancer cells. Altered energy metabolism is a hallmark of cancer and has been shown to play a critical role in tumor initiation and development (18). The effects of NAMPT on glycolysis in HBV-expressing liver cancer cells were assessed in the present study. The inactivation of NAMPT by FK866 resulted in a markedly decreased ECAR during the stages of glycolysis following glucose and oligomycin injection, whereas co-treatment with NMN partially rescued the glycolytic capacity of HepG2.2.15 and HepAD38 cells (Fig. 4A). In addition, FK866 suppressed glucose uptake, lactate production and ATP levels, and these effects were also reversed by NMN (Fig. 4B-D). Since PKM2 and LDHA are required for lactate production and GLUT1 is directly associated with glucose uptake, whether FK866 affected the expression of these glycolysis-associated genes was investigated. The results indicated that the mRNA and protein expression levels of GLUT1, PKM2 and LDHA were decreased following FK866 treatment in HepG2.2.15 and HepAD38 cells (Fig. 4E and F), and co-treatment with NMN markedly attenuated the FK866-induced effects on the levels of GLUT1, PKM2 and LDHA (Fig. 4E and F). The enzymatic activity of PKM2 in HepG2.2.15 and HepAD38 cells was observed to be reduced in response to FK866 treatment, and this reduction in activity was diminished by NMN (Fig. 4G). A similar result was noted in the enzymatic activity of LDHA (Fig. 4H).

Further experiments were conducted using HepG2.2.15 and HepAD38 cells transfected with specific siRNA targeting 
A

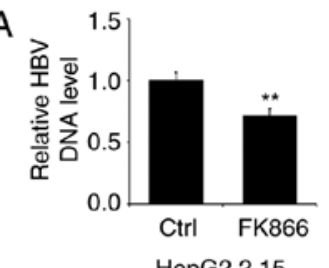

HepG2.2.15

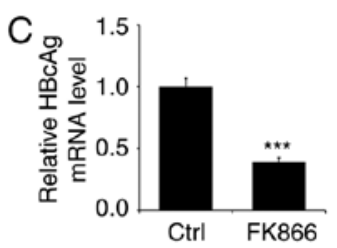

HepG2.2.15

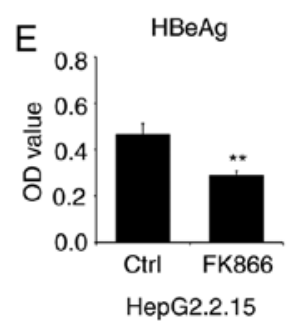

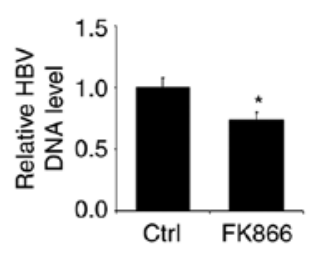

HepAD38

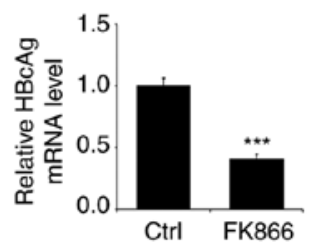

HepAD38

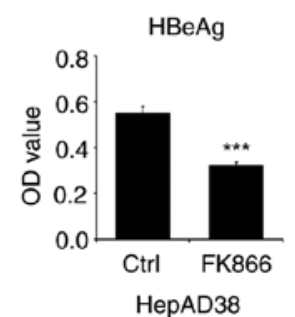

B

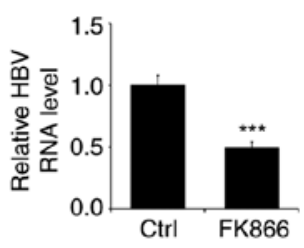

HepG2.2.15

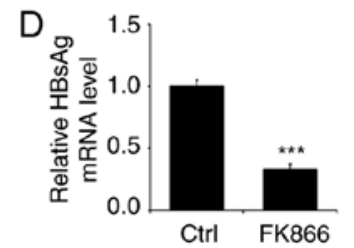

HepG2.2.15

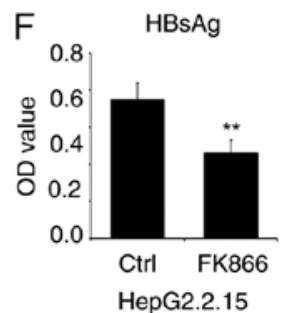

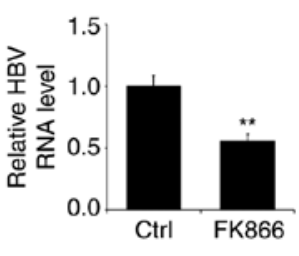

HepAD38

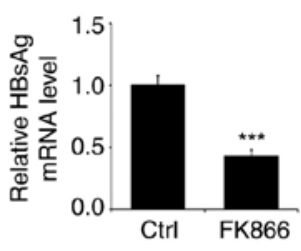

HepAD38

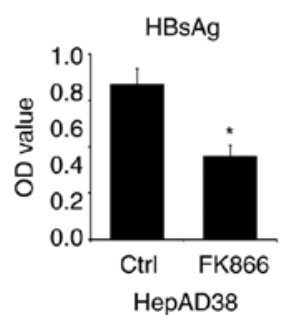

Figure 2. Inhibition of NAMPT by FK866 represses HBV replication and transcription. HepG2.2.15 and HepAD38 cells were treated with 20 nM FK866 for $24 \mathrm{~h}$. (A) The number of HBV DNA copies relative to those of untreated cells in the cell-free culture media were measured by qPCR analysis. (B) The relative levels of HBV RNA, (C) HBcAg mRNA and (D) HBsAg mRNA were detected by RT-qPCR analysis. The levels of secreted (E) HBeAg and (F) HBsAg in the culture media were measured by ELISA. ${ }^{*} \mathrm{P}<0.05,{ }^{* *} \mathrm{P}<0.01,{ }^{* * *} \mathrm{P}<0.001$. NAMPT, nicotinamide phosphoribosyltransferase; HBV, hepatitis B virus; HBcAg, HBV core antigen; HBsAg, HBV surface antigen; RT-qPCR, reverse transcriptase-quantitative PCR; Ctrl, control; OD, optical density.

A

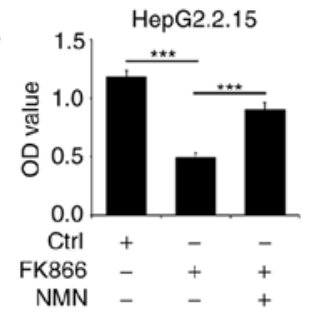

C

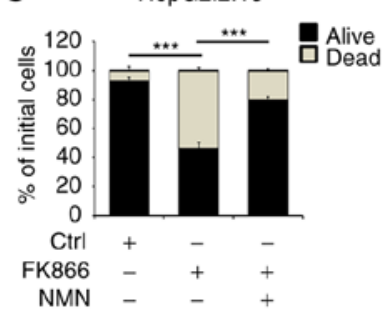

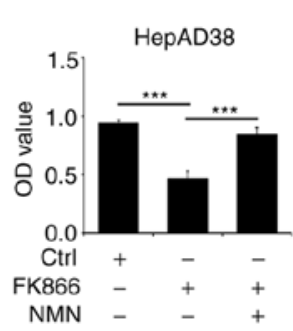

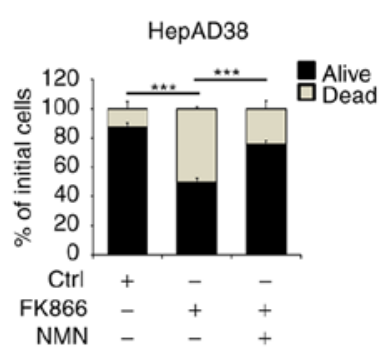

B

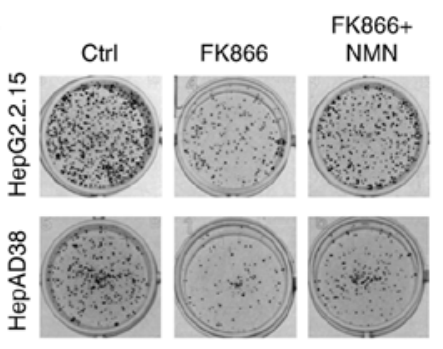

D

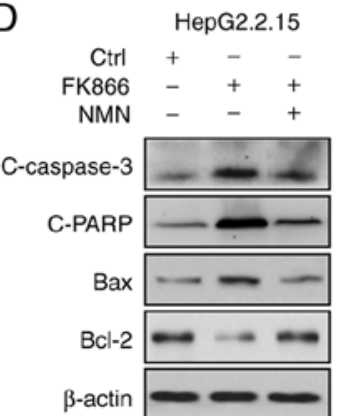

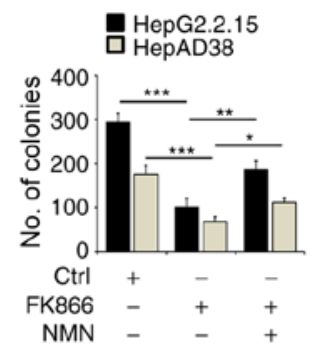

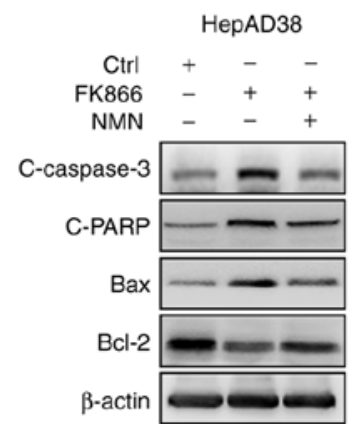

Figure 3. FK866-induced NAMPT inhibition decreases cell survival and promotes cell death in HBV-expressing liver cancer cells. HepG2.2.15 and HepAD38 cells were treated with FK866 alone or in combination with NMN for $24 \mathrm{~h}$. (A) Cell viability was measured by CCK-8 assay, (B) a colony formation assay was performed and (C) cell death was determined by the trypan blue exclusion assay. (D) The treated HepG2.2.15 and HepAD38 cells were also subjected to immunoblot analysis for the detection of apoptosis-associated proteins. ${ }^{*} \mathrm{P}<0.05,{ }^{* *} \mathrm{P}<0.01,{ }^{* * *} \mathrm{P}<0.001$. NAMPT, nicotinamide phosphoribosyltransferase; HBV, hepatitis B virus; NMN, nicotinamide mononucleotide; CCK-8, Cell Counting Kit-8; C, cleaved; PARP, poly (ADP-ribose) polymerase; Ctrl, control; OD, optical density.

NAMPT (Fig. 5A). The cells transfected with siNAMPT displayed decreased cell viability and increased cell death compared with those transfected with siNC (Fig. 5B and C). In addition, the knockdown of NAMPT inhibited glycolytic 

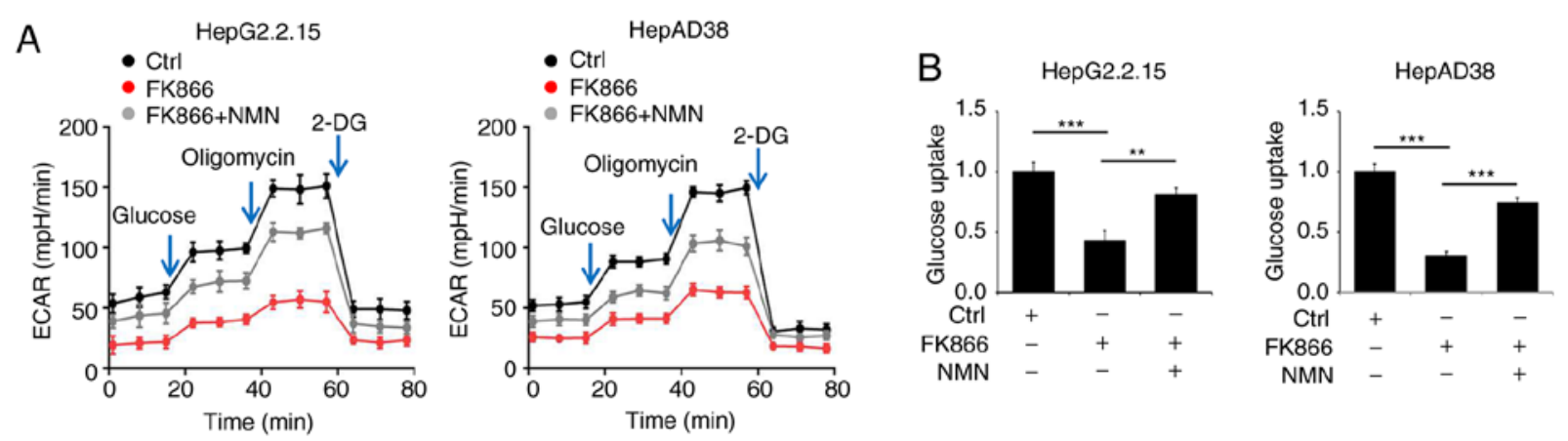

C
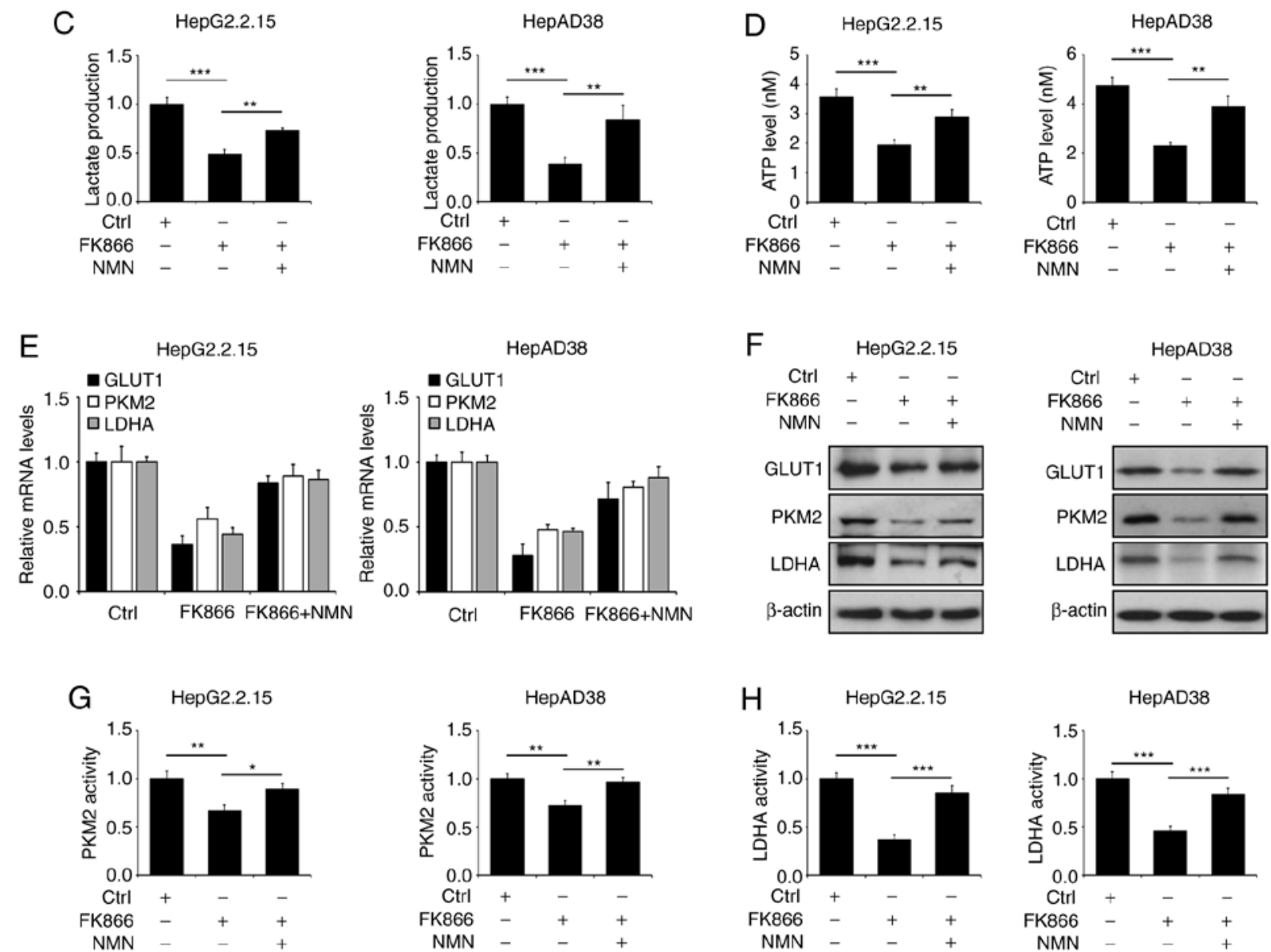

Figure 4. NAMPT inactivation impairs aerobic glycolysis in HBV-expressing liver cancer cells. HepG2.2.15 and HepAD38 cells were treated with FK866 alone or in combination with NMN for $24 \mathrm{~h}$. (A) The ECAR, (B) glucose uptake, (C) lactate production and (D) ATP levels of the cells were evaluated. (E) The levels of GLUT1, PKM2 and LDHA mRNA relative to those in untreated controls were detected by RT-qPCR analysis. (F) Immunoblot analysis was performed to detect the expression of GLUT1, PKM2 and LDHA, and the enzymatic activity of (G) PKM2 and (H) LDHA was determined. "P<0.05, ${ }^{* *} \mathrm{P}<0.01,{ }^{* * *} \mathrm{P}<0.001$. NAMPT, nicotinamide phosphoribosyltransferase; HBV, hepatitis B virus; NMN, nicotinamide mononucleotide; ECAR, extracellular acidification rate; GLUT1, glucose transporter 1; PKM2, pyruvate kinase M2; LDHA, lactate dehydrogenase A; RT-qPCR, reverse transcriptase-quantitative PCR; Ctrl, control; 2-DG, 2-deoxy-D-glucose.

capacity, glucose uptake and lactate production in HepG2.2.15 and HepAD38 cells (Fig. 5D-F). These data suggest that NAMPT regulates energy metabolism by enhancing aerobic glycolysis in HBV-expressing liver cancer cells.

\section{Discussion}

HBV is an enveloped DNA virus with four overlapping genes and can cause acute and chronic infections. Chronic infection with HBV contributes to chronic inflammation of the liver, leading to liver impairment, liver cirrhosis and eventually the development of liver cancer (19). Therefore, chronic HBV infection is a major risk factor for liver cancer. HBV utilizes the cellular machinery of the host for viral DNA replication, protein production and packaging. $\mathrm{HBx}$ is a non-structural protein of the HBV genome, which contributes to the development of liver cancer by modulating various cellular signaling pathways to enhance HBV replication and transcription (20). A recent study indicated that a C-terminal-truncated $\mathrm{HBx}$ protein promoted hepatocarcinogenesis via activation of the caveolin-1/low-density lipoprotein receptor-related protein 6/FERM domain containing 5 axis (21). HBx has also be 
A
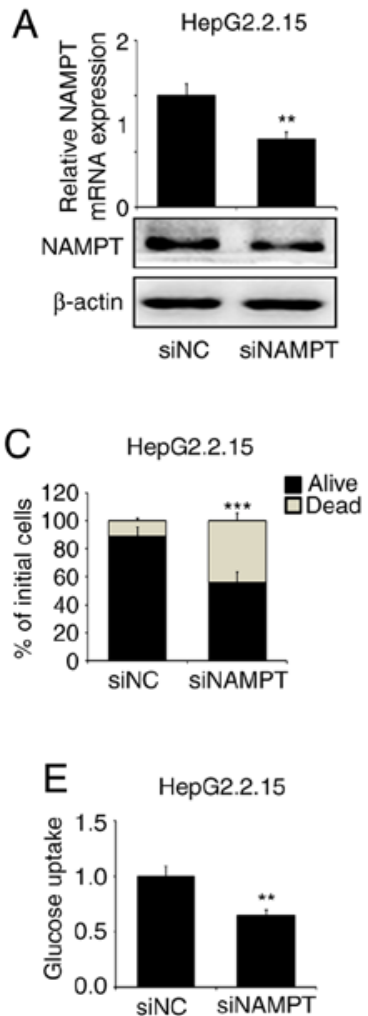
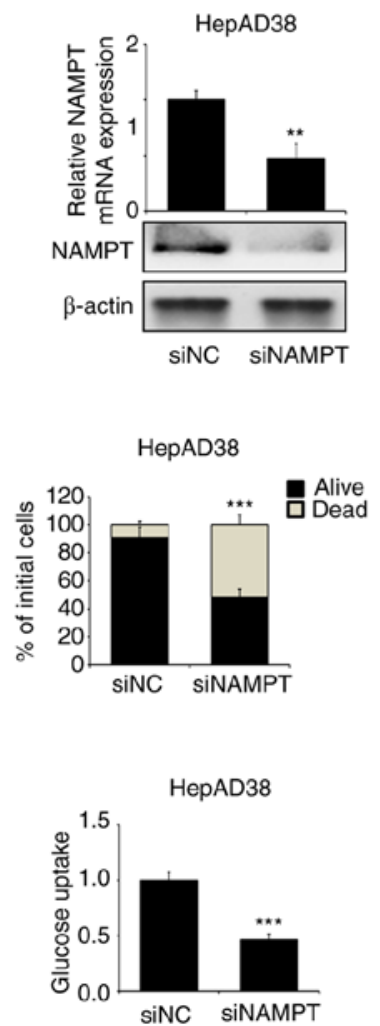
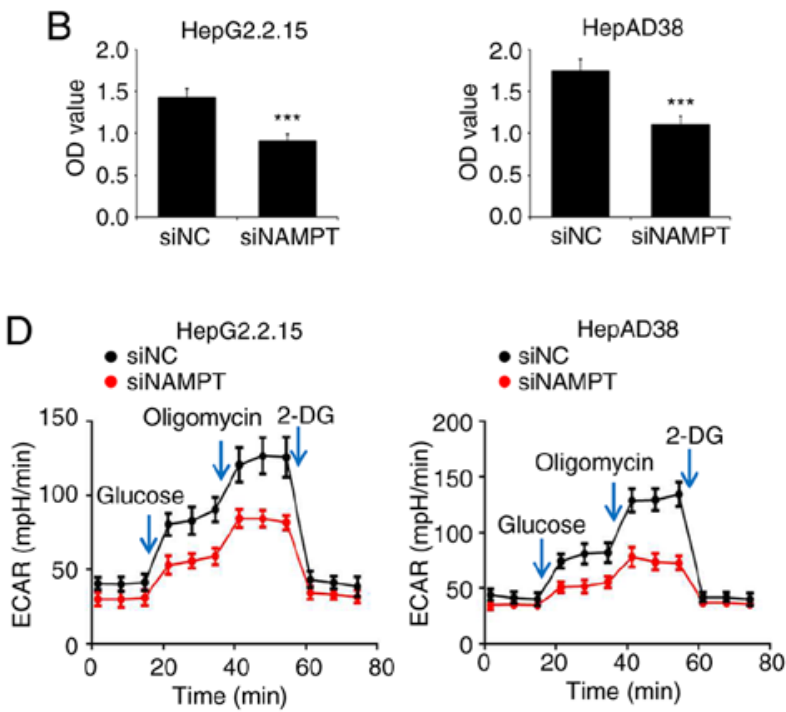

F

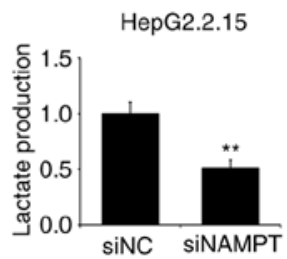

HepAD38

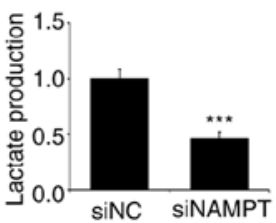

Figure 5. NAMPT suppression diminishes cell growth and aerobic glycolysis in HBV-expressing liver cancer cells. HepG2 and HepG2.2.15 cells were transfected with siRNA targeting NAMPT or siNC. (A) RT-qPCR and western blot analyses of NAMPT expression in the cells. (B) Cell viability was measured using the CCK- 8 assay and (C) cell death was determined by the trypan blue exclusion assay. (D) The ECAR was determined, and (E) glucose uptake and (F) lactate production assays were performed. ${ }^{* *} \mathrm{P}<0.01,{ }^{* * *} \mathrm{P}<0.001$. NAMPT, nicotinamide phosphoribosyltransferase; HBV, hepatitis $\mathrm{B}$ virus; RT-qPCR, reverse transcriptase-quantitative PCR; siRNA, small interfering RNA; siNAMPT, siRNA targeting NAMPT; siNC, negative control siRNA; CCK-8, Cell Counting Kit-8; ECAR, extracellular acidification rate; OD, optical density.

shown to maintain the proliferation of liver cancer cells via the microRNA-155-mediated zinc fingers and homeoboxes 2 gene silencing (22). In addition, $\mathrm{HBx}$ has been found to be localized in the lumen of the endoplasmic reticulum (ER) where it interacts with $78-\mathrm{kDa}$ glucose-regulated protein, leading to a reduction in ER stress and DNA repair that prevents liver cancer cells from undergoing apoptosis (23). However, the role of NAMPT in HBV-associated liver cancer progression is currently unclear.

In the present study, the data indicated that the mRNA and protein expression levels of NAMPT were higher in HBV-positive liver cancer cells than in HBV-negative liver cancer cells. Moreover, the HBx-expressing HepG2 and Huh-7 cells had higher NAMPT levels than the corresponding untransfected liver cancer cells. These data imply that HBx promotes the expression of NAMPT. The inhibition of NAMPT by FK866 significantly diminished the secretion of HBV DNA and the mRNA expression levels of HBV RNA, HBcAg and HBsAg, suggesting a critical role of NAMPT in HBV replication. Furthermore, FK866 also reduced the secretion of $\mathrm{HBeAg}$ and HBsAg by HBV-producing liver cancer cells. Therefore, the data suggest the existence of a positive feedback loop between NAMPT and HBV replication. HBV infection has been shown to cause metabolic reprogramming in host cells to generate the energy and molecular components required for their replication and activation $(24,25)$. For example, a study reported that the levels of metabolic intermediates, including phenylalanine, L-glutamic acid and serine, were significantly elevated in the serum of patients with HBV compared with healthy controls (26). In addition, another study demonstrated that HBV replication accelerated the biosynthesis of hexosamine and phosphatidylcholine, leading to the dysregulated expression of glycolytic enzymes and increased secretion of lactate (27). In the present study, the data indicated that the FK866-induced inactivation of NAMPT inhibited glycolysis and the glycolytic capacity of HBV-expressing liver cancer cells. In addition, the expression levels and enzymatic activities of PKM2 and LDHA, which are key regulators of glucose metabolism, were decreased in response to FK866. These results indicate an important role of NAMPT in the regulation of glucose metabolic reprogramming, whereas the disruption of NAD metabolism may be considered a potential strategy for cancer therapy. The inhibition of NAMPT by FK866 resulted in a substantial reduction of cell viability and increased cell death in HBV-expressing liver cancer cells, whereas the supplementation of NMN partially reversed the effect of FK866. The present study further supports the essential function of NAMPT in HBV-mediated liver malignancy.

Accumulating evidence suggests that oxidative stress is necessary for HBV-mediated liver cancer development and progression. For example, HBV infection promotes an intracellular accumulation of reactive oxygen species that is necessary for HBV replication in host cells $(28,29)$. Increased oxidative stress leads to elevated levels of DNA damage, which 
activates poly (ADP-ribose) polymerase (PARP)-mediated repair. As PARP-1 consumes NAD, which is the substrate for its activation (30), HBV-positive liver cancer cells may become sensitive to NAD deficiency triggered by NAMPT inhibition. Furthermore, sirtuins are a family of NAD-dependent deacetylases that have been shown to regulate numerous biological functions, including lipid and energy metabolism, DNA damage, oxidative stress and cell growth, survival and death (31-33). Sirtuin 6 promotes the transcription and replication of HBV by upregulating the expression of peroxisome proliferator-activated receptor $\alpha$ (34). In addition, sirtuin 1 has been shown to be essential for HBV-associated liver cancer tumorigenesis (35-37). Therefore, the reduction of NAD synthesis by NAMPT inhibition may be an effective and promising method for the treatment of patients with $\mathrm{HBV}$-associated liver cancer.

In conclusion, the findings of the present study provide the first evidence that HBV promotes NAMPT expression, and indicate that the activation of NAMPT is positively associated with HBV replication and transcription in liver cancer cells. The knockdown of NAMPT repressed cell growth and promoted cell death in HBV-positive liver cancer cells. Moreover, the inactivation of NAMPT suppressed glucose uptake, lactate production and ATP levels, suggesting a critical role of NAMPT in the regulation of aerobic glycolysis. Based on this evidence, the present study suggests an important molecular mechanism by which NAMPT promotes HBV replication and HBV-mediated cell growth through the manipulation of glucose metabolic reprogramming. In addition, it highlights NAMPT as a promising target for the therapy of patients with $\mathrm{HBV}$-associated liver cancer.

\section{Acknowledgements}

Not applicable.

\section{Funding}

The study was supported by the Development and Regeneration Key Laboratory of Sichuan Province, Chengdu Medical College (grant no. SYS20-05), the National Undergraduate Training Program for Innovation and Entrepreneurship (grant no. S202013705006) and the Research Fund of Chengdu Medical College (grant no. CYZ19-12).

\section{Availability of data and materials}

All data generated or analyzed during this study are included in this published article.

\section{Authors' contributions}

CFM and ZS conceived the study. HJG, HYL and ZHC performed the experiments, and WJZ, JJL, JYZ and JW helped to conduct the experiments. CFM and HJG wrote the manuscript. ZS, XYL and TZ interpreted the data and revised the manuscript. CFM and ZS supervised the study. CFM and ZS confirm the authenticity of all the raw data. All authors read and approved the final manuscript.

\section{Ethics approval and consent to participate}

Not applicable.

\section{Patient consent for publication}

Not applicable.

\section{Competing interests}

The authors declare that they have no competing interests.

\section{References}

1. Siegel R, Ma J, Zou Z and Jemal A: Cancer statistics, 2014. CA Cancer J Clin 64: 9-29, 2014.

2. El-Serag HB and Rudolph KL: Hepatocellular carcinoma: Epidemiology and molecular carcinogenesis. Gastroenterology 132: 2557-2576, 2007.

3. World Health Organization: Guidelines for the Prevention, Care and Treatment of Persons with Chronic Hepatitis B Infection, 2015.

4. Levrero M and Zucman-Rossi J: Mechanisms of HBV-induced hepatocellular carcinoma. J Hepatol 64 (1 Suppl): S84-S101, 2016.

5. Xu QG, Yuan SX, Tao QF, Yu J, Cai J, Yang Y, Guo XG, Lin KY, Ma JZ, Dai DS, et al: A novel HBx genotype serves as a preoperative predictor and fails to activate the JAK1/STATs pathway in hepatocellular carcinoma. J Hepatol 70: 904-917, 2019.

6. Xiao W, Wang RS, Handy DE and Loscalzo J: NAD $(\mathrm{H})$ and NADP $(H)$ redox couples and cellular energy metabolism. Antioxid Redox Signal 28: 251-272, 2018.

7. Yaku K, Okabe K and Nakagawa T: NAD metabolism: Implications in aging and longevity. Ageing Res Rev 47: 1-17, 2018.

8. Garten A, Schuster S, Penke M, Gorski T, de Giorgis T and Kiess W: Physiological and pathophysiological roles of NAMPT and NAD metabolism. Nat Rev Endocrinol 11: 535-546, 2015.

9. Sampath D, Zabka TS, Misner DL, O'Brien T and Dragovich PS: Inhibition of nicotinamide phosphoribosyltransferase (NAMPT) as a therapeutic strategy in cancer. Pharmacol Ther 151: 16-31, 2015.

10. Lee J, Kim H, Lee JE, Shin SJ, Oh S, Kwon G, Kim H, Choi YY, White MA, Paik S, et al: Selective cytotoxicity of the NAMPT inhibitor FK866 toward gastric cancer cells with markers of the epithelial-mesenchymal transition, due to loss of NAPRT. Gastroenterology 155: 799-814 e13, 2018.

11. Espindola-Netto JM, Chini CCS, Tarrago M, Wang E, Dutta S, Pal K, Mukhopadhyay D, Sola-Penna M and Chini EN: Preclinical efficacy of the novel competitive NAMPT inhibitor STF-118804 in pancreatic cancer. Oncotarget 8: 85054-85067, 2017.

12. Gehrke I, Bouchard ED, Beiggi S, Poeppl AG, Johnston JB, Gibson SB and Banerji V: On-target effect of FK866, a nicotinamide phosphoribosyl transferase inhibitor, by apoptosis-mediated death in chronic lymphocytic leukemia cells. Clin Cancer Res 20: 4861-4872, 2014.

13. Mo CF, Li J, Yang SX, Guo HJ, Liu Y, Luo XY, Wang YT, Li MH, Li JY and Zou Q: IQGAP1 promotes anoikis resistance and metastasis through Rac1-dependent ROS accumulation and activation of Src/FAK signalling in hepatocellular carcinoma. Br J Cancer 123: 1154-1163, 2020.

14. Xu J, Liu H, Chen L, Wang S, Zhou L, Yun X, Sun L, Wen Y and $\mathrm{Gu}$ J: Hepatitis B virus $X$ protein confers resistance of hepatoma cells to anoikis by up-regulating and activating p21-activated kinase 1. Gastroenterology 143: 199-212.e4, 2012.

15. Li K, Mo C, Gong D, Chen Y, Huang Z, Li Y, Zhang J, Huang L, Li Y, Fuller-Pace FV, et al: DDX17 nucleocytoplasmic shuttling promotes acquired gefitinib resistance in non-small cell lung cancer cells via activation of $\beta$-catenin. Cancer Lett 400: 194-202, 2017.

16. Livak JK and Schmittgen Td: Analysis of relative gene expression data using quantitative Pcr and the 2(-delta delta $\mathrm{c}(\mathrm{T})$ ) method. Methods 25: 402-408, 2001. 
17. Li K, Gao B, Li J, Chen H, Li Y, Wei Y, Gong D, Gao J, Zhang J, Tan W, et al: ZNF32 protects against oxidative stress-induced apoptosis by modulating C1QBP transcription. Oncotarget 6: 38107-38126, 2015.

18. Hanahan D and Weinberg RA: Hallmarks of cancer: The next generation. Cell 144: 646-674, 2011.

19. Ringelhan M, O'Connor T, Protzer U and Heikenwalder M: The direct and indirect roles of $\mathrm{HBV}$ in liver cancer: Prospective markers for HCC screening and potential therapeutic targets. J Pathol 235: 355-367, 2015.

20. Buendia MA and Neuveut C: Hepatocellular carcinoma. Cold Spring Harb Perspect Med 5: a021444, 2015.

21. Mao X, Tey SK, Ko FCF, Kwong EML, Gao Y, Ng IO, Cheung ST, Guan XY and Yam JWP: C-terminal truncated HBx protein activates caveolin-1/LRP6/ $\beta$-catenin/FRMD5 axis in promoting hepatocarcinogenesis. Cancer Lett 444: 60-69, 2019.

22. Song X, Tan S, Wu Z, Xu L, Wang Z, Xu Y, Wang T, Gao C, Gong Y, Liang X, et al: HBV suppresses ZHX2 expression to promote proliferation of HCC through miR-155 activation. Int $\mathbf{J}$ Cancer 143: 3120-3130, 2018.

23. Li J, He J, Fu Y, Hu X, Sun LQ, Huang Y and Fan X: Hepatitis $B$ virus $X$ protein inhibits apoptosis by modulating endoplasmic reticulum stress response. Oncotarget 8: 96027-96034, 2017.

24. Pallett LJ, Gill US, Quaglia A, Sinclair LV, Jover-Cobos M, Schurich A, Singh KP, Thomas N, Das A, Chen A, et al: Metabolic regulation of hepatitis $B$ immunopathology by myeloid-derived suppressor cells. Nat Med 21: 591-600, 2015.

25. Fisicaro P, Boni C, Barili V, Laccabue D and Ferrari C: Strategies to overcome HBV-specific T cell exhaustion: Checkpoint inhibitors and metabolic re-programming. Curr Opin Virol 30: 1-8, 2018.

26. Gao R, Cheng J, Fan C, Shi X, Cao Y, Sun B, Ding H, Hu C, Dong $F$ and Yan X: Serum metabolomics to identify the liver disease-specific biomarkers for the progression of hepatitis to hepatocellular carcinoma. Sci Rep 5: 18175, 2015.

27. Li H, Zhu W, Zhang L, Lei H, Wu X, Guo L, Chen X, Wang Y and Tang $\mathrm{H}$ : The metabolic responses to hepatitis $\mathrm{B}$ virus infection shed new light on pathogenesis and targets for treatment. Sci Rep 5: 8421, 2015.
28. Schinzari V, Barnaba V and Piconese S: Chronic hepatitis B virus and hepatitis $C$ virus infections and cancer: Synergy between viral and host factors. Clin Microbiol Infect 21: 969-974, 2015.

29. Ganesan M, Eikenberry A, Poluektova LY, Kharbanda KK and Osna NA: Role of alcohol in pathogenesis of hepatitis B virus infection. World J Gastroenterol 26: 883-903, 2020.

30. Ying W, Alano CC, Garnier P and Swanson RA: NAD+ as a metabolic link between DNA damage and cell death. J Neurosci Res 79: 216-223, 2005.

31. Roulston A and Shore GC: New strategies to maximize therapeutic opportunities for NAMPT inhibitors in oncology. Mol Cell Oncol 3: e1052180, 2016.

32. Griffiths HBS, Williams C, King SJ and Allison SJ: Nicotinamide adenine dinucleotide $\left(\mathrm{NAD}^{+}\right)$: Essential redox metabolite, co-substrate and an anti-cancer and anti-ageing therapeutic target. Biochem Soc Trans 48: 733-744, 2020.

33. Yaku K, Okabe K, Hikosaka K and Nakagawa T: NAD metabolism in cancer therapeutics. Front Oncol 8: 622, 2018.

34. Jiang H, Cheng ST, Ren JH, Ren F, Yu HB, Wang Q, Huang AL and Chen J: SIRT6 inhibitor, OSS_128167 restricts hepatitis B virus transcription and replication through targeting transcription factor peroxisome proliferator-activated receptors $\alpha$. Front Pharmacol 10: 1270, 2019.

35. Ren JH, Tao Y, Zhang ZZ, Chen WX, Cai XF, Chen K, Ko BC, Song CL, Ran LK, Li WY, et al: Sirtuin 1 regulates hepatitis $\mathrm{B}$ virus transcription and replication by targeting transcription factor AP-1. J Virol 88: 2442-2451, 2014.

36. Pant K, Mishra AK, Pradhan SM, Nayak B, Das P, Shalimar D, Saraya A and Venugopal SK: Butyrate inhibits HBV replication and HBV-induced hepatoma cell proliferation via modulating SIRT-1/Ac-p53 regulatory axis. Mol Carcinog 58: 524-532, 2019.

37. Wang Q, Cheng ST and Chen J: HBx mediated increase of SIRT1 contributes to HBV-related hepatocellular carcinoma tumorigenesis. Int J Med Sci 17: 1783-1794, 2020.

This work is licensed under a Creative Commons Attribution-NonCommercial-NoDerivatives 4.0 International (CC BY-NC-ND 4.0) License. 